Article

\title{
Influence of Horseback Riding and Horse Simulator Riding on Heart Rate Variability: Are There Differences?
}

\author{
Santos Villafaina ${ }^{1}\left(\mathbb{D}\right.$, Carmen Cordón-González ${ }^{2}$, Daniel Collado-Mateo ${ }^{1,3, * \mathbb{C} \text {, }}$ \\ Juan P. Fuentes-García ${ }^{2, *(D)}$, J. C. Adsuar ${ }^{2}$ D, E. Merellano-Navarro ${ }^{3}$ and J. A. Parraca ${ }^{4,5}$ \\ 1 Physical Activity and Quality of Life Research Group (AFYCAV), Faculty of Sport Science, \\ University of Extremadura, 10003 Cáceres, Spain; svillafaina@unex.es \\ 2 Faculty of Sport Science, University of Extremadura, 10003 Cáceres, Spain; \\ ccordong@alumnos.unex.es (C.C.-G.); jadssal@unex.es (J.C.A.) \\ 3 Facultad de Educación, Universidad Autónoma de Chile, Talca 1670, Chile; emerellano@gmail.com \\ 4 Departamento de Desporto e Saúde, Escola de Ciência e Tecnologia, Universidade de Évora, \\ 7000-727 Évora, Portugal; jparraca@uevora.pt \\ 5 Comprehensive Health Research Center (CHRC), University of Évora, 7000-727 Évora, Portugal \\ * Correspondence: dcolladom@unex.es (D.C.-M.); jpfuent@unex.es (J.P.F.-G.); Tel.: +34-927-25-74-60 (J.P.F.-G.)
}

Received: 25 April 2019; Accepted: 27 May 2019; Published: 29 May 2019

\begin{abstract}
This study aimed to compare the heart rate variability (HRV) in healthy young people while riding a real horse or a horse gait simulator. The sample consisted of a group of 23 healthy young adults aged 22.91 (2.37), who rode a horse for five minutes at walking speed and spent five minutes on a horse gait simulator, while their HRV values were being recorded. Furthermore, immediately after each protocol, the HRV at rest was also recorded to observe the acute effects. We used the paired samples t-test to compare between the HRV during the horse-riding and the horse simulator-riding activities, as well as the differences in the acute effects between both situations. The findings indicate that the HRV was lower when participants were riding the horse compared with the activity on the horse simulator. However, no differences were observed immediately after the two protocols. Therefore, we state that the sympathetic tone is higher while riding a real horse than while riding a horse simulator. These differences may be due to emotional aspects and not due to differences in the physical load, considering the absence of differences in the acute effects.
\end{abstract}

Keywords: horseback; heart rate variability; emotions; physical load

\section{Introduction}

Horseback riding provides a dynamic base of support, making it an excellent therapeutic tool for improving the balance and the trunk strength [1], as well as for working with special populations [2-5]. The horse's movement could lead to benefits in psychological, social, and physical welfare, involving effects on many human body systems, such as the sensorial, musculoskeletal, limbic, vestibular, and ocular [6].

Horse gait simulators have been developed to reduce costs and improve accessibility. This tool makes this therapy more adaptable and accessible to subjects [7], allowing indoor exercise [8]. However, it is suggested that these devices may be limited exclusively to physical and muscular benefits, but perhaps do not include the mental and cognitive aspects influenced by the direct interaction with the animal-in this case, the horse [8].

Most studies have focused on assessing physical benefits, but as far as we know, only two $[9,10]$ have evaluated the differences between these two kinds of activities at the autonomic nervous system 
level. In this sense, the heart rate variability (HRV) is a non-invasive index of the autonomous nerve activity, which reports the balance of the autonomic nervous system based on the successive heartbeats (also called the RR interval) variation over an interval of time [11]. The cardiovascular center, located in the medulla of the brainstem, adjusts the heart rate via shifting the balance between the sympathetic and the parasympathetic nervous systems. A reduced HRV is associated with an increased risk of death from several causes [12], since it reflects a reduced regulatory capacity to adapt to challenges such as exercise or stressors [13]. In the same line, HRV is an indicator of resilience, behavior flexibility, or cognitive load [14-16], increasing the activation of the parasympathetic nervous system and decreasing that of the sympathetic nervous system.

Previous studies have shown how physical activity, emotions, or stress can cause changes in the HRV [17]. Regarding horseback riding, it caused a more pronounced sympathetic tone than the simulator gait training $[9,10]$. These differences were detected during $30 \mathrm{~min}$ of activities in the study by Matsuura et al. [9] and 11 min plus eight jumps in the study by Ille et al. [10]. Hence, there is no evidence of the effect of a short single bout ( $5 \mathrm{~min}$ ) on the HRV.

This research aims to study the differences in terms of HRV in two situations-riding a horse at a walking speed and riding a simulator of the horse's gait—both during the activity and immediately after it.

\section{Methods}

\subsection{Participants}

Before the enrollment, the sample size was calculated taking into account the reported data from the study by Ille et al. (10) and the differences regarding HRV (RMSSD index) between horseback riding and a simulator. Taking into account the output of PASS 11 software, a sample size of 17 participants was enough to achieve $96 \%$ of power to detect significant differences ( $p$-value $<0.05$ ). Finally, in order to improve the statistical power, a convenience sample of 23 subjects participated in this study. All of them were voluntary university students who were enrolled in the university facilities, males $(n=17)$ and females $(n=6)$, with an average age of 22.91 (2.37) and a fear of horseback riding of $1.65(1.19)$ measured by a visual analogue scale (VAS) where 0 indicated the total absence of fear and 10 indicated the maximum fear possible.

Inclusion criteria for participants were:

- Not be taking medication affecting the nervous system.

- Not suffering from any heart or neurodegenerative disease.

- Have ridden a horse before.

Participants were also advised not to engage in vigorous physical exercise, drink coffee, or take any other substance that could affect the nervous system $24 \mathrm{~h}$ before undergoing the protocol.

Protocols were approved by the University Bioethics Committee (reference number: 89/2018), and the informed consent was collected from all the participants before starting the study. The protocol did not harm any of the subjects or animals participating in the study. There was no incentive for participation in the study.

\subsection{Experimental Design and Procedures}

A cross-sectional study was conducted on a single group of individuals. They underwent the following two protocols: (1) riding a horse at walking speed (a horse's lowest gait speed) for five minutes, and (2) riding a horse gait simulator for five minutes (at the lowest pace allowed by the device). The order was randomized in order to control the potential influence of one task on the other. The HRV was recorded during the two conditions following the recommendations of the European Society of Cardiology [13]. Immediately after carrying out each of the two tests (simulator and horse), a five-minute record at rest was registered to evaluate the acute effects of these activities on the HRV. 


\subsection{Instruments and Data Processing}

The study was conducted in the equestrian infrastructures at the "Heredade da Mitra" and horses provided by the Equestrian Association from Évora. This association has an agreement with the University of Évora.

The model TA-022 (Horse riding machine, Zhejiang, China) was used to simulate the horse's gait. The simulator has four speed levels and a random one. The lowest speed level was used for the present study, simulating the horse's walk.

The HRV was measured during and immediately after the two experimental activities according to the recommendations of the Task Force of the European Society of Cardiology and the North American Society of Pacing and Electrophysiology [13] during a short five-minute sitting record and also following recommendations from previous studies [14,15]. The HRV was recorded through a heart rate monitor (Polar RS800CX, Finland), whose reliability has been proven [16]. This wireless device was placed on the chest of participants, allowing a comfortable and non-limiting HRV register. The Kubios HRV software (v. 2.1) [17] was used to process and extract the data. A middle filter was applied to correct possible artifacts, identifying those RR intervals that are shorter/longer than $0.25 \mathrm{~s}$, compared to the average among previous beats. Correction replaces the identified artifacts by cubic spline interpolation.

In this study, different HRV variables were studied: (a) time domain, such as HR mean, standard deviation of the entire RR interval (SDNN), NN50 count divided by the total number of all the NN ranges (Pnn50), and the square root of the sum mean of the differences squares between adjacent NN intervals (rMSSD); (b) frequency domain, including the low-frequency (LF) relation (LF, 0.04 to 0.15 Hertz)/high frequency (HF, 0.15-0.4 Hertz) (LF/HF) and total capacity; (c) non-linear measures, such as entropy (Approximate Entropy [ApEn] and Sample Entropy [SampEn]).

\subsection{Statistical Analysis}

Parametric statistical analyses were used according to the results of the Shapiro-Wilks and Kolmogorov-Smirnov tests.

HRV differences between riding a horse and riding a gait simulator were evaluated using paired sample t-tests. These statistical tests were also used to evaluate the acute effects in HRV immediately after the execution of each of the protocols. A $p$-value lower than 0.05 was considered significant.

The statistical analysis was carried out with the statistical package SPSS (Statistical Package for Social Sciences, version 25.0 for Windows).

\section{Results}

Table 1 shows the results of the paired sample t-test comparing the HRV during the two protocols: riding a horse or riding a simulator. In this regard, significant differences were observed among the time domain variables (HRmean, RR, SDNN, PNN50, and RMSSD), frequency domain (Total Power), and non-linear variables (SampEn). We can see that the HR is higher when the subjects ride on the horse compared with on the simulator (95.20 [14.51]). Conversely, the HRV-related variables (RR, SDNN, PNN50, RMSSD, total capacity, and SampEn) reached significantly lower values when the participants were riding a real horse (Table 1 ).

Table 2 shows the comparison between the acute effects (five minutes immediately after the activities) between the horse riding and the simulator riding. There were no statistically significant differences in the studied variables between the two proposed situations. 
Table 1. Heart rate variability values during the horse riding and the horse simulator riding.

\begin{tabular}{ccccc}
\hline Variable & Horse Mean (SD) & Simulator Mean (SD) & $p$-Value & Cohen's D \\
\hline MeanHR & $95.20(14.51)$ & $83.19(11.74)$ & $<0.001$ & 0.874 \\
RR & $650.75(99.8)$ & $739.95(105.83)$ & $<0.001$ & -0.863 \\
SDNN & $34.39(14.40)$ & $44.71(12,40)$ & $<0.001$ & -0.764 \\
pNN50 & $7.29(8.01)$ & $12.69(10.46)$ & 0.001 & -0.548 \\
RMSSD & $25.18(13.39)$ & $33.32(14.07)$ & $<0.001$ & -0.591 \\
HF & $22.00(10.59)$ & $21.95(11.22)$ & 0.984 & 0.004 \\
LF & $77.91(10.64)$ & $77.97(11.25)$ & 0.981 & -0.005 \\
LF/HF & $4.73(2.87)$ & $4.74(2.78)$ & 0.982 & -0.005 \\
Total Power & $1483.11(1219)$ & $2224.82(1315)$ & 0.005 & -0.583 \\
ApEn & $1.11(0.98)$ & $1.10(0.81)$ & 0.573 & 0.113 \\
SampEn & $1.35(0.25)$ & $1.48(0.23)$ & 0.029 & -0.549 \\
\hline
\end{tabular}

HR: Heart Rate; RR: Time between R-R intervals; SDNN: Standard deviation of all normal to normal RR intervals; pNN50: Intervals percentage $>50 \mathrm{~ms}$ different from preceding interval; RMSSD: The square root of the squares mean of successive RR interval differences; LF/HF: Ratio of Low Frequency (LF) $\left(\mathrm{ms}^{2}\right)$ / High Frequency (HF) $\left(\mathrm{ms}^{2}\right)$;

Total Power: The sum of all spectra; ApEn: Approximate Entropy; SampEn: Sample Entropy.

Table 2. Acute effects analysis of riding a horseback or in the simulator.

\begin{tabular}{ccccc}
\hline Variable & Horse Mean (SD) & Simulator Mean (SD) & $p$-Value & Cohen's D \\
\hline MeanHR & $74.28(10.85)$ & $74.42(9.44)$ & 0.887 & -0.014 \\
RR & $836.00(126.57)$ & $830.30(109.72)$ & 0.611 & 0.046 \\
SDNN & $65.47(23.61)$ & $69.09(21.55)$ & 0.083 & -0.156 \\
pNN50 & $31.61(18.72)$ & $30.11(16.25)$ & 0.431 & 0.083 \\
RMSSD & $59.10(28.58)$ & $57.70(25.10)$ & 0.591 & 0.050 \\
HF & $36.24(16.05)$ & $31.95(16.87)$ & 0.353 & 0.261 \\
LF & $63.68(16.06)$ & $67.98(16.87)$ & 0.354 & -0.261 \\
LF/HF & $2.48(2.11)$ & $3.17(2.45)$ & 0.198 & -0.300 \\
Total Capacity & $5865.04(3640)$ & $5161.86(3877)$ & 0.602 & -0.079 \\
ApEn & $1.09(0.09)$ & $1.06(0.10)$ & 0.162 & 0.262 \\
SampEn & $1.61(0.28)$ & $1.54(0.23)$ & 0.118 & 0.301 \\
\hline
\end{tabular}

HR: Heart Rate; RR: Time between R-R intervals; SDNN: Standard deviation of all normal to normal RR intervals; pNN50: Intervals percentage $>50 \mathrm{~ms}$ different from preceding interval; RMSSD: The square root of the squares mean of successive RR interval differences; LF/HF: Ratio of Low Frequency (LF) $\left(\mathrm{ms}^{2}\right) /$ High Frequency (HF) $\left(\mathrm{ms}^{2}\right)$;

Total Power: The sum of all spectra; ApEn: Approximate Entropy; SampEn: Sample Entropy.

\section{Discussion}

The present study aimed to compare the HRV in healthy subjects during and immediately after riding a horse and riding a horse gait simulator. The main finding was that the HRV was significantly lower while riding on horseback than during the activity in the simulator. Conversely, the acute effects on the HRV of these two tasks did not significantly differ. Given that the HRV may be sensitive to exercise intensity in the following minutes after exercise performance [18], we hypothesize that both tasks caused similar physical demands.

The results show that the sympathetic tone is higher when riding a real horse compared with the protocol on the horse simulator, which is supported by previous studies $[9,10]$. However, the protocol for these two works takes longer duration ( $30 \mathrm{~min}$ in the study by Matsuura et al. [9] and $11 \mathrm{~min}$ plus eight jumps in the study by Ille et al. [10]. Therefore, differences in autonomic modulation during a short single bout of five minutes had never been detected. This is interesting not only in healthy people, but also in special populations where sometimes it is extremely difficult (above all at the beginning of intervention programs) to perform long horseback riding sessions.

The sympathetic response in the heart rate is mediated by the release of epinephrine and norepinephrine $[19,20]$. However, not only can exercise induce the release of these substances [21], but emotions can also influence this process [22]. In this regard, the HRV is also affected by emotions [23]. Accordingly, the differences observed are probably the result of emotions rather than the physical 
load. Previous studies already went in that direction, studying the increase in HRV caused by physical activities with high emotional load, such as parachute jumps [24] or comparing the effects on the HRV of riding a simulator or a horse [9]. This last study found an increase in the parasympathetic activity two hours after riding the horse, which may be related to a compensatory relaxing effect. Therefore, and along with our results, we can state that although a riding simulator can provide a similar physical load to a horse movement, this type of equipment could not completely replace a horse, since they cannot induce similar emotions compared with real animals [25]; also, the simulator only oscillates in a programmed pattern, while a real horse, as a living being, provides a complex oscillation to the rider [26]. Future studies should consider this statement and increase the recording time by a few hours in order to identify the point at which sympathetic and parasympathetic activity change after exercise performance, as well as the role of emotions in this type of activity. In this line, future studies should examine the emotional experience of the participants during both simulator and horseback riding conditions.

Previous research studies have examined the role of animals in therapy and education, stating the benefits of animal-assisted therapy [25], or the motivating effect caused by an animal in the educational environment [27]. Simulators have also been shown to have positive effects as horseback-riding substitutes [28], promoting therapeutic benefits associated with postural control and muscle strength, including low cost, as well as greater safety and accessibility [29]. However, mental or psychological effects appear to be more limited in simulators compared to animal-assisted therapies [8,30]. The current manuscript supports the absence of psychological benefits since the physiological response seems to be different despite the similar physical load. This result could indicate the importance of emotions during the performance of physical activities.

Our study also has some limitations. First, the sample was composed of healthy young adults, which limited the possibility of extrapolating the results to other pathological or special populations. Second, no emotional questionnaire was given to participants, which may limit the interpretation of the HRV parameters.

\section{Conclusions}

Horseback riding leads to a decrease in the HRV in most of the HRV variables studied when compared to the same activity performed with a horse gait simulator. However, their acute effects did not significantly differ, which may indicate that the physical load of both tasks was similar. In this sense, the differences observed may be due to emotional aspects. Therefore, although simulators are able to produce similar physical demands, they may fail at eliciting similar emotional responses in the rider and also at reproducing the complex gait of a living horse.

Author Contributions: J.C.A., S.V., J.A.P. and D.C.-M. conceived the study. C.C.-G., E.M.-N., J.A.P., J.C.A., S.V. and D.C.-M collected the data. C.C.-G., D.C.-M., J.P.F.-G., E.M.-N. and S.V. analysed the data. J.P.F.-G., J.C.A., J.P.F.-G. and D.C.-M. designed Figures and Tables. C.C.-G., S.V., J.A.P. and D.C.-M. wrote the manuscript. E.M.-N, J.P.F.-G., J.A.P., J.C.A. and D.C.-M. provided critical revisions on the successive drafts. All authors approved the manuscript in its final form.

Funding: UÉvora-UniverCIDADE IV program. (Instituto Português do Desporto e Juventude-I.P., Apoio à Atividade Desportiva 2018, Contrato-Programa de Desenvolvimento Desportivo n. CP/605/DD/2018).

Acknowledgments: The authors appreciate the support of Paulo Duque Fonseca and Lourenço Machado. Also, the author SV is supported by a grant from the regional department of economy and infrastructure of the Government of Extremadura and European Social Fund (PD16008). The funders had no role in the study design, data collection and analysis, decision to publish, or preparation of the manuscript.

Conflicts of Interest: The authors declare no competing interests. 


\section{References}

1. Lechner, H.E.; Kakebeeke, T.H.; Hegemann, D.; Baumberger, M. The effect of hippotherapy on spasticity and on mental well-being of persons with spinal cord injury. Arch. Phys. Med. Rehabil. 2007, 88, 1241-1248. [CrossRef]

2. Benda, W.; McGibbon, N.H.; Grant, K.L. Improvements in muscle symmetry in children with cerebral palsy after equine-assisted therapy (hippotherapy). J. Altern. Complement. Med. 2003, 9, 817-825. [CrossRef]

3. Dirienzo, L.N.; Dirienzo, L.T.; Baceski, D.A. Heart rate response to therapeutic riding in children with cerebral palsy: An exploratory study. Pediatr. Phys. Ther. 2007, 19, 160-165. [CrossRef] [PubMed]

4. Kaiser, L.; Smith, K.A.; Heleski, C.R.; Spence, L.J. Effects of a therapeutic riding program on at-risk and special education children. J. Am. Vet. Med Assoc. 2006, 228, 46-52. [CrossRef]

5. Seow, L.L.; Toh, C.G.; Wilson, N.H. Strain measurements and fracture resistance of endodontically treated premolars restored with all-ceramic restorations. J. Dent. 2015, 43, 126-132. [CrossRef]

6. Koca, T.T.; Ataseven, H. What is hippotherapy? The indications and effectiveness of hippotherapy. North. Clin. Istanb. 2015, 2, 247-252. [CrossRef]

7. Kijima, R.; Kouno, M.; Hashimoto, K.; Jiang, Y.; Aoki, T.; Ojika, T. (Eds.) Karakuri Horse Riding Therapy. In Proceedings of the 8th International Conference on Rehabilitation Robotics (ICORR 2003), Daejeon, Korea, 22-25 April 2003; KAIST: Daejeon, Korea, 2003.

8. Hilliere, C.; Collado-Mateo, D.; Villafaina, S.; Duque-Fonseca, P.; Parraca, J.A. Benefits of Hippotherapy and Horse Riding Simulation Exercise on Healthy Older Adults: A Systematic Review. PM R J. Inj. Funct. Rehabil. 2018, 10, 1062-1072. [CrossRef] [PubMed]

9. Matsuura, A.; Nagai, N.; Funatsu, A.; Irimajiri, M.; Yamazaki, A.; Hodate, K. Comparison of the Short-Term Effects of Horse Trekking and Exercising with a Riding Simulator on Autonomic Nervous Activity. Anthrozoös 2011, 24, 65-77. [CrossRef]

10. Ille, N.; von Lewinski, M.; Aurich, C.; Erber, R.; Wulf, M.; Palme, R.; Greenwood, B.; Aurich, J. Riding Simulator Training Induces a Lower Sympathetic Response in Riders Than Training with Horses. J. Equine Vet. Sci. 2015, 35, 668-672. [CrossRef]

11. Thayer, J.F.; Ahs, F.; Fredrikson, M.; Sollers, J.J., III; Wager, T.D. A meta-analysis of heart rate variability and neuroimaging studies: Implications for heart rate variability as a marker of stress and health. Neurosci. Biobehav. Rev. 2012, 36, 747-756. [CrossRef] [PubMed]

12. Routledge, F.S.; Campbell, T.S.; McFetridge-Durdle, J.A.; Bacon, S.L. Improvements in heart rate variability with exercise therapy. Can. J. Cardiol. 2010, 26, 303-312. [CrossRef]

13. Camm, A.J.; Malik, M.; Bigger, J.T.; Breithardt, G.; Cerutti, S.; Cohen, R.J.; Coumel, P.; Fallen, E.L.; Kennedy, H.L.; Kleiger, R.E.; et al. Heart rate variability. Standards of measurement, physiological interpretation, and clinical use. Eur. Heart J. 1996, 17, 354-381.

14. Sánchez-Molina, J.; Robles-Pérez, J.J.; Clemente-Suárez, V.J. Assessment of psychophysiological response and specific fine motor skills in combat units. J. Med. Syst. 2018, 42, 67. [CrossRef]

15. Beltrán-Velasco, A.I.; Bellido-Esteban, A.; Ruisoto-Palomera, P.; Clemente-Suárez, V.J. Use of Portable Digital Devices to Analyze Autonomic Stress Response in Psychology Objective Structured Clinical Examination. J. Med. Syst. 2018, 42, 35. [CrossRef]

16. da Costa, M.P.; da Silva, N.T.; de Azevedo, F.M.; Pastre, C.M.; Marques Vanderlei, L.C. Comparison of Polar((R)) RS800G3 heart rate monitor with Polar((R)) S810i and electrocardiogram to obtain the series of RR intervals and analysis of heart rate variability at rest. Clin. Physiol. Funct. Imaging 2016, 36, 112-117.

17. Tarvainen, M.P.; Niskanen, J.-P.; Lipponen, J.A.; Ranta-aho, P.O.; Karjalainen, P.A. Kubios HRV-Heart rate variability analysis software. Comput. Methods Programs Biomed. 2014, 113, 210-220. [CrossRef] [PubMed]

18. Michael, S.; Jay, O.; Halaki, M.; Graham, K.; Davis, G.M. Submaximal exercise intensity modulates acute post-exercise heart rate variability. Eur. J. Appl. Physiol. 2016, 116, 697-706. [CrossRef]

19. Trautwein, W.; Kameyama, M. Intracellular control of calcium and potassium currents in cardiac cells. Jpn. Heart J. 1986, 27, 31-50.

20. Brown, H.F.; DiFrancesco, D.; Noble, S.J. How does adrenaline accelerate the heart? Nature 1979, 280, $235-236$. [CrossRef]

21. Warren, J.B.; Dalton, N.; Turner, C.; Clark, T.J.; Toseland, P.A. Adrenaline secretion during exercise. Clin. Sci. 1984, 66, 87-90. [CrossRef] [PubMed] 
22. Strange, B.A.; Dolan, R.J. Beta-adrenergic modulation of emotional memory-evoked human amygdala and hippocampal responses. Proc. Natl. Acad. Sci. USA 2004, 101, 11454-11458. [CrossRef]

23. Choi, K.H.; Kim, J.; Kwon, O.S.; Kim, M.J.; Ryu, Y.H.; Park, J.E. Is heart rate variability (HRV) an adequate tool for evaluating human emotions?-A focus on the use of the International Affective Picture System (IAPS). Psychiatry Res. 2017, 251, 192-196. [CrossRef]

24. Sanchez-Molina, J.; Robles-Perez, J.J.; Clemente-Suarez, V.J. Effect of Parachute Jump in the Psychophysiological Response of Soldiers in Urban Combat. J. Med. Syst. 2017, 41, 99. [CrossRef]

25. Beetz, A.; Uvnäs-Moberg, K.; Julius, H.; Kotrschal, K. Psychosocial and psychophysiological effects of human-animal interactions: The possible role of oxytocin. Front. Psychol. 2012, 3, 234. [CrossRef] [PubMed]

26. Matsuura, A.; Maruta, H.; Iwatake, T.; Kumagai, T.; Nakanowatari, T.; Hodate, K. The beneficial effects of horse trekking on autonomic nervous activity in experienced rider with no disability. Anim. Sci. J./Nihon Chikusan Gakkaiho 2017, 88, 173-179. [CrossRef]

27. Diamond, A.; Lee, K. Interventions shown to aid executive function development in children 4 to 12 years old. Science 2011, 333, 959-964. [CrossRef] [PubMed]

28. Kim, M.J.; Kim, T.; Choi, Y.; Oh, S.; Kim, K.; Yoon, B. The effect of a horse riding simulator on energy expenditure, enjoyment, and task difficulty in the elderly. Eur. J. Integr. Med. 2016, 8, 723-730. [CrossRef]

29. Han, J.Y.; Kim, J.M.; Kim, S.K.; Chung, J.S.; Lee, H.C.; Lim, J.K.; Lee, J.; Park, K.Y. Therapeutic effects of mechanical horseback riding on gait and balance ability in stroke patients. Ann. Rehabil. Med. 2012, 36, 762-769. [CrossRef]

30. Rigby, B.R.; Papadakis, Z.; Bane, A.A.; Park, J.K.; Grandjean, P.W. Cardiorespiratory and biomechanical responses to simulated recreational horseback riding in healthy children. Res. Q. Exerc. Sport 2015, 86, 63-70. [CrossRef] [PubMed]

(C) 2019 by the authors. Licensee MDPI, Basel, Switzerland. This article is an open access article distributed under the terms and conditions of the Creative Commons Attribution (CC BY) license (http://creativecommons.org/licenses/by/4.0/). 